\title{
REAL-TIME ESTIMATION OF MEAN ARTERIAL BLOOD PRESSURE
}

\author{
Eleonora Zanderigo* Daniel Leibundgut ${ }^{* *}$ \\ Franta Kraus* Rolf Wymann ** Manfred Morari* \\ * Automatic Control Laboratory, Swiss Federal Institute of \\ Technology (ETH), CH-8092 Zurich, Switzerland \\ zanderigo, kraus, morari@control.ee.ethz.ch \\ ** University Hospital (Inselspital), CH-3010 Bern, \\ Switzerland, leibundgut, wymann@dkf. unibe.ch
}

\begin{abstract}
A real-time non-invasive method for mean arterial blood pressure (MAP) estimation is presented. MAP is estimated by effectively combining two components: one describing the low frequency behaviour and one the fast variations of MAP. The method, developed on 20 volunteers under anaesthesia allows for statistically significant improvement with respect to the standard cuff system. This approach represents a major improvement to surgical routine by providing more information about the patient's haemodynamic state without requiring additional sensors. Further, it allows for the use of automatic MAP control also in those routine operations where only the standard non-invasive MAP monitoring is applied. Copyright ${ }^{\circledR} 2005$ IFAC.
\end{abstract}

Keywords: modelling, biomedical systems, identification, signal correlation, medical applications.

\section{INTRODUCTION}

Blood pressure is a patient's vital parameter and is therefore constantly monitored during surgery or intensive care. Mean arterial blood pressure (MAP) is related to the haemodynamic state of the patient and various events such as blood loss, surgical stimulation or drug infusions can lead to MAP changes during surgery. A strong external painful stimulation, i.e. skin incision by the surgeon, triggers the autonomic stress response of the patient, which leads to fast MAP and heart rate (HR) increases. This reaction of the body to external painful stimulation represents one of the most important causes of blood pressure changes in the surgical theater.

During surgery the patient is anaesthetized. A balanced anaesthesia can be defined as a reversible pharmacological state where the patient's muscle relaxation, analgesia and hypnosis are guaranteed. Thus, a fundamental component of anaesthesia is the suppression of pain and consequently of patient's reactions to the painful surgical stimulation. However, since pain is a highly subjective feeling, no specific measurement of pain is available for unconscious patients. The International Association for the Study of Pain defines pain as an unpleasant sensory and emotional experience associated with actual or potential tissue damage. Thus, it may even be improper to speak about pain during general anaesthesia when the subject is unconscious (Petersen-Felix et al., 1998). Therefore, the intraoperative administration of opiates is not directly related to pain treatment (Habibi and Coursin, 1996), but to the suppression of the patient's autonomic stress reactions to surgical stimulation, which must be minimized during 
surgery (Prys-Roberts et al., 1990). In this sense, continuous control of blood pressure represents an end point for balanced anaesthesia. The different existing methods to acquire MAP can be divided into two main categories: invasive and noninvasive methods. The invasive method consists of a cannula inserted into the arteria radialis. This procedure, however, is expensive, time consuming and poses risks of trauma and infections for the patient. Thus, in order to contain the occurrence of complications and the cost of treatment, non-invasive (oscillometric) measurement of blood pressure is generally preferred to the insertion of an intra-arterial catheter, whenever an invasive monitoring is not required for specific clinical reasons. Different methods of non-invasive blood pressure monitoring already exist, some of them deliver a continuous beat-to-beat measurement (Yamakoshi et al., 1980; Mackay, 1964). All these methods, however, require additional equipment and are sensitive to the positioning of the sensor. Therefore, the simple cuff system is still the most widely used non-invasive blood pressure monitoring device.

Due to physical limitations of the device and patient's comfort, the standard sampling time for the oscillometric determination of blood pressure is relatively high (5 $\mathrm{min})$. This sampling time is too long compared to the frequency of the fast blood pressure variations caused by the autonomic stress reaction. Due to this mismatch the system is not capable of keeping track of the important fast variations in the MAP signal, leading to a considerable lack of information.

A robust and reliable estimation of blood pressure during two consecutive non-invasive measurements would cover the lack of information caused by the long sampling time of the device. Having a continuous estimation of the blood pressure signal would represent a major improvement, providing the anesthetist continuous blood pressure information, while keeping the stress for the patient, risks and costs minimal. Further, it would represent a key step in the development of automatic control of blood pressure. In anaesthesia different control loops already exist to control, for example, depth of hypnosis (Gentilini et al., 2001), muscle relaxation (Stadler et al., 2003) or MAP (Gentilini et al., 2002). However, up to now it has not been possible to use the MAP controller when only noninvasive MAP measurements are available, which still represent the standard clinical procedure.

Various attempts to relate blood pressure to other physiological signals already exist (Allen and Murray, 1999; Gulcur and Bahadirlar, 1998; Pinna et al., 1996). An estimation method based on pulse arrival time as additional signal has also been published (Chen et al., 2000). The method, however, suffers from long time lags and is therefore not applicable to on-line estimation.

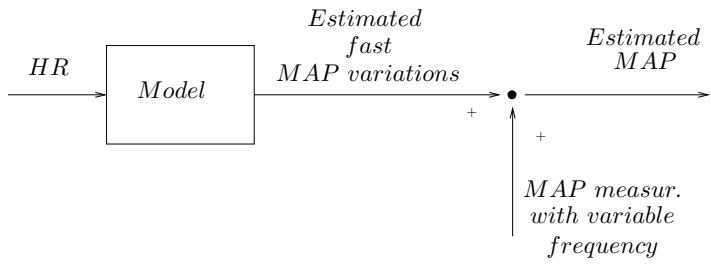

Fig. 1. Estimation algorithm

Unlike MAP, HR is always acquired at high frequency. The aim of our investigation was to evaluate whether additional information about the high frequency behaviour of MAP could be derived from a routinely available signal such as HR and whether, despite inter-patient variability, an efficient algorithm could be developed to reliably estimate MAP, allowing us to non-invasively monitor MAP in real time without requiring to add sensors to the standard cuff system.

\section{METHODS}

The data used for the development of the method were obtained from a volunteer study. A detailed description of the study design is given in the appendix. For each patient two sets of data were available: the HR signal and the invasively recorded MAP signal, referred to as the "true data". From the true MAP signal, two signals were constructed to mimic the intermittent noninvasive measurements. Two different sampling methods were considered:

- constant sampling frequency of 5 min;

- variable sampling frequency, according to the following algorithm: starting from a sampling time of $5 \mathrm{~min}$, if the difference in two subsequent measurements exceeds $\pm 7 \mathrm{mmHg}$, the sampling time is decreased to $2.5 \mathrm{~min}$ and re-set to $5 \mathrm{~min}$ if the difference between two subsequent measurements with sampling time $2.5 \mathrm{~min}$ is lower than $\pm 7 \mathrm{mmHg}$.

The MAP signals obtained in this manner are referred to as "MAP measurements" with constant resp. with variable sampling rate. The hypothesis to be tested is that MAP can be estimated by combining two components: MAP measurements with variable sampling rate, keeping track of the slow variations in MAP, and an estimation of fast MAP variations based on $\mathrm{HR}$, as shown in Fig. 1. The so obtained signal is referred to as the estimated MAP.

The relationship between HR variations and MAP variations was postulated to be linear and modelled by a discrete ARX model structure:

$$
\begin{aligned}
y(t) & +a_{1} \cdot y(t-1)+\ldots+a_{n_{a}} \cdot y\left(t-n_{a}\right)= \\
& =b_{n_{k}} \cdot u\left(t-n_{k}\right)+b_{n k+1} \cdot u\left(t-n_{k}-1\right)+ \\
& +b_{n_{k}+n_{b}} \cdot u\left(t-n_{k}-n_{b}\right)+e(t)
\end{aligned}
$$


The hypothesis test consists of two main steps: estimation of model order and coefficients and performance assessment by comparison of the estimated MAP signal with the true data, as described in the following paragraphs.

\subsection{Parameter Estimation}

The identification of model order and coefficients was based on the true data, acquired at $128 \mathrm{~Hz}$ during the volunteer study. The true HR and MAP signals were preprocessed to remove outliers, mean and trends. Both signals were filtered through a 3rd order band-pass Butterworth filter with a low cut-off frequency of $0.0003 \mathrm{~Hz}$ and a high cut-off frequency of $0.003 \mathrm{~Hz}$, according to the frequency range of the fast variations chosen to be investigated. The sampling time adopted for the ARX model was 10 sec. For each volunteer, half of the data available were used as identification and half as validation data set. The model coefficients were determined through a single least squares (LS) estimation procedure on the whole identification data set. The model estimated in this way allows to estimate the fast variations of MAP on the basis of the HR signal. The input signal to the model is the HR signal, the output is an estimate of the fast variations of MAP, which added to the MAP measurements with variable sampling rate generates the estimated MAP. The estimated MAP to be compared with the true data was generated starting from a HR signal with a sampling time of 10 sec.

Since the order of the linear model to be used was not known a priori, different model orders were tested by repetition of the estimation procedure. Models with number of coefficients $\left(n_{a}\right.$ and $\left.n_{b}\right)$ varying from 1 to 5 and a delay $\left(n_{k}\right)$ of 0 or 1 were estimated and their performance distribution among the volunteers was evaluated as described in the following section. The best order was then selected as the one having the highest mean performance among the volunteers.

So far, the model was assumed to be generally valid for all volunteers. However, inter-patient variability represents a fundamental issue in developing biological models. Not always is it possible to identify a generally valid model able to adequately describe the behaviour of all subjects without requiring individual estimation of the parameters. Thus, in addition to the described identification of the generalized model over the whole volunteer set, a best model was estimated for each of the considered subjects with "individualized" model order and parameter values. These models are referred to as the "individualized models".

\subsection{Performance Assessment}

For validation of the method, the estimated MAP was compared with the true MAP and performance of the estimation was assessed by Pearson correlation coefficients (CC), root mean square of estimation errors (RMSE) and fit. This comparison was performed for every volunteer on the corresponding validation data, so that a distribution of CC, RMSE and fit among the volunteers was obtained. The estimation errors are defined as:

$$
e_{\text {estim }}=100 \cdot \frac{(\hat{y}-y)}{y}
$$

where $\hat{y}$ is the estimated value of the considered variable $y$. The fit defines the percentage of output variations around its mean, which could be described by the model, according to the standard fit definition in system identification (Ljung, 1999):

$$
f i t=100 \cdot\left(1-\frac{\|\hat{y}-y\|}{\|y-\bar{y}\|}\right)
$$

where $\hat{y}$ is the estimated value of the considered variable $\mathrm{y}$ and $\bar{y}$ its mean value. Note that the fit can also take negative values, meaning that the estimate of the signal is worse than the one obtained by just averaging it.

In order to assess the performance improvement attributable to the estimation of fast variations, the performance of the estimation was compared with that of the measurement with variable sampling rate alone. In addition, to evaluate the importance of inter-patient variability, the performance for the individualized and the generalized models were compared.

\section{RESULTS}

\subsection{Parameter Estimation}

Mean observation time per patient was $266 \mathrm{~min}$, ranging from 227 to $302 \mathrm{~min}$. Volunteers No. 10 and No. 15 presented an almost flat MAP over the duration of the study despite external stimulation and were therefore excluded from the analysis.

The highest mean correlation coefficient criterium, the highest mean fit criterium and the minimum mean RMSE criterium all led to the identification of the same model order as the best one. For this model order the LS-estimates of model coefficients and the delay are reported in Table 1.

\subsection{Performance Assessment}

For the best performing model, the performance indicators are summarized in Table 2. The fit 
Table 1. LS coefficients estimation for the best performing model.

\begin{tabular}{cc}
\hline$a_{1}$ & -0.9995 \\
$b_{1}$ & 1.0626 \\
$b_{2}$ & -2.5289 \\
$b_{3}$ & 2.2326 \\
$b_{4}$ & -0.7689 \\
$n_{k}$ & 1 \\
\hline
\end{tabular}

Table 2. Performance indicators among the volunteers for the best performing model.

\begin{tabular}{cccc}
\hline Vol.No. & Fit(\%) & CC & RMSE(\%) \\
\hline 1 & 40.5 & 0.81 & 4.99 \\
2 & 48.04 & 0.87 & 6.24 \\
3 & 39.18 & 0.83 & 4.62 \\
4 & 56.60 & 0.90 & 3.79 \\
5 & 66.02 & 0.94 & 4.68 \\
6 & 65.43 & 0.94 & 5.34 \\
7 & 66.99 & 0.95 & 4.68 \\
8 & 74.83 & 0.97 & 4.20 \\
9 & 72.44 & 0.96 & 4.64 \\
10 & - & - & - \\
11 & 41.65 & 0.87 & 6.16 \\
12 & 60.40 & 0.92 & 5.81 \\
13 & 71.18 & 0.96 & 4.25 \\
14 & 61.52 & 0.92 & 5.58 \\
15 & - & - & - \\
16 & 65.28 & 0.94 & 3.95 \\
17 & 67.39 & 0.95 & 4.10 \\
18 & 64.14 & 0.93 & 3.59 \\
19 & 66.54 & 0.95 & 4.87 \\
20 & 73.08 & 0.96 & 4.12 \\
mean & 61.18 & 0.92 & 4.76 \\
sd & 11.40 & 0.05 & 0.80 \\
\hline
\end{tabular}

distribution among the volunteers ranged from $39.18 \%$ to $74.83 \%$ with a mean value of $61.18 \%$ and a standard deviation of $11.4 \%$. The correlation coefficients between estimated and actual values of MAP ranged from 0.81 to 0.97 among the volunteers with a mean value of 0.92 and a standard deviation of 0.05 . The RMSE values varied from $3.59 \%$ to $6.24 \%$ with a mean value of $4.76 \%$ and a standard deviation of $0.8 \%$.

Fig. 2 shows the different fit distributions obtained by measurements with constant sampling rate (a), measurements with variable sampling rate (b) and estimated MAP (c). Performance improvement between the latter two cases was statistically assessed by a paired t-test $(p=0.0003)$.

The results of the performance comparison between the generalized and the individualized models is reported in Fig. 3. The fit of the individualized models reached from $39.61 \%$ to $76.03 \%$ with a mean value of $61.95 \%$ and a standard deviation of $11.5 \%$. The statistical significance of the accuracy loss caused by neglecting inter-patient variability was evaluated by a paired t-test $(p=0.077)$.

Fig. 4 and 5 show the signal evolution of true and measured MAP, estimate MAP and HR for two volunteers.

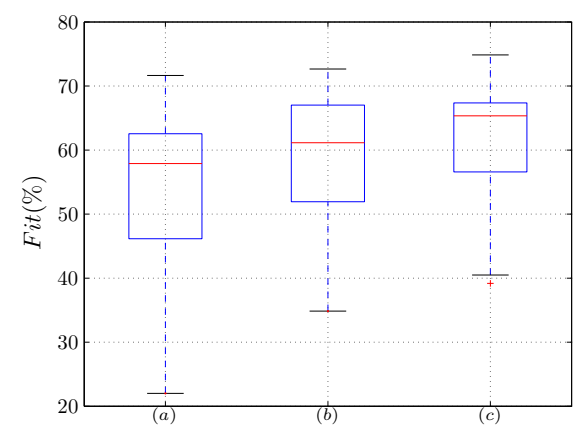

Fig. 2. Fit between true MAP and (a) measured MAP with constant sampling rate (b), measured MAP with variable sampling rate and (c) estimated MAP. Distribution among the volunteers.

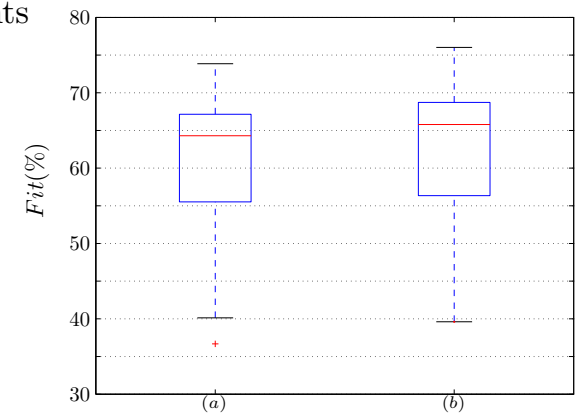

Fig. 3. Fit between true and estimated MAP obtained by (a) the generalized model or (b) the individualized models. Distribution among the volunteers.

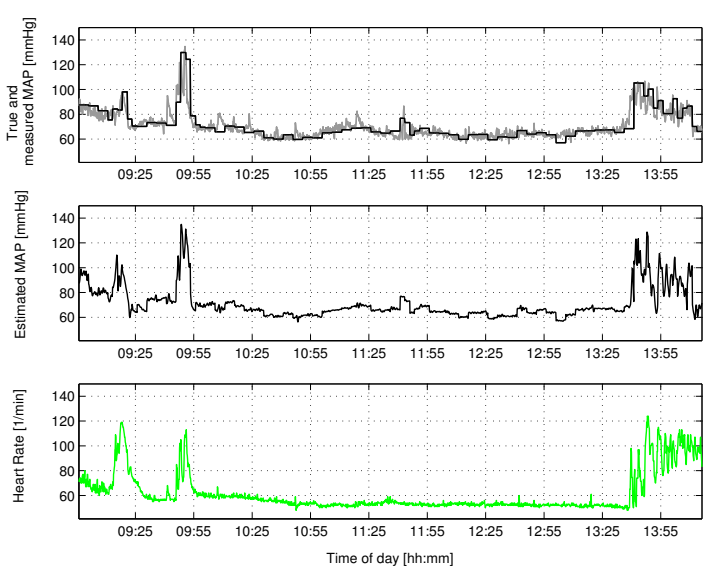

Fig. 4. Temporal signal evolution of true and measured MAP with variable sampling rate (top), estimated MAP (middle) and HR (bottom) for volunteer No.11.

\section{DISCUSSION}

The limit of the non-invasive cuff measurement device lies in its high sampling interval that doesn't allow for tracking of fast blood pressure changes. Fast blood pressure changes, however, represent an important factor during surgery, since they are linked to the autonomic stress reaction of the patient, which has to be minimized through intraoperative opiates infusion. 

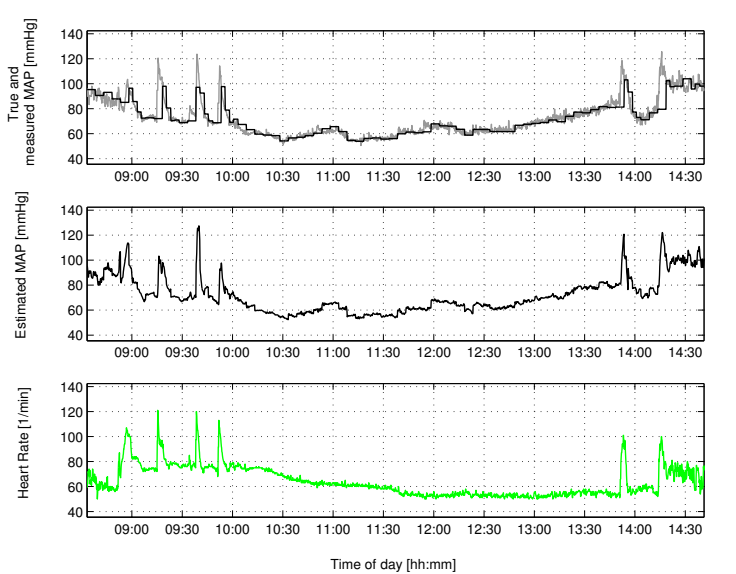

Fig. 5. Temporal signal evolution of true and measured MAP with variable sampling rate (top), estimated MAP (middle) and HR (bottom) for volunteer No.14.

The standard sampling time of 5 min used in clinical practice is not the minimum allowed by physical considerations, so a simple increase in the sampling frequency of the device could already result in a performance increase. In order to perform a significant evaluation of the improvement achieved through the estimation procedure proposed, the performance of the estimation method was compared to the performance of an improved cuff device. In the improved cuff device the sampling frequency is increased according to a variable sampling rate algorithm, still respecting the limits imposed physical limitations and patient's comfort. The sampling rate algorithm varies the measurement frequency adaptively depending on the entity of the changes between consecutive measurements, allowing for a performance increase with respect to the standard cuff measurements with constant sampling rate, as shown in Fig. 2 (a)-(b). The remaining performance lack is represented by those variations of even higher frequency that cannot be covered by simply increasing the sampling frequency further. Thus, it represents the information lack addressed by our estimation procedure.

In the volunteer study, external painful stimulation triggered the patient's autonomic stress reaction. This reaction leads to both blood pressure and heart rate increases. Therefore, it is reasonable to assume that the fast changes due to stress reaction in MAP and HR could be related. However, there is no reason to assume that the same should hold also for the slow variations. Therefore, additional measurements of the low frequency component are still needed in order to track the whole signal evolution of MAP.

Application of anaesthetics and analgesics clearly influences patient's reactions to painful stimuli. In order to take this into account, drug dosages and administration patterns were changed between experiments as well as during each single experi- ment, so that the model derived can be assumed to be valid over a broad range of infused drug concentrations.

The estimated MAP obtained by combining the low frequency measurement with variable sampling rate and the estimation of high frequency components shows a high correlation with the true MAP signal over the validation data set, confirming that the proposed method leads to a reliable estimation of MAP. Further, the estimation of fast variations leads to a a statistically significant improvement in the performance, as shown in Fig. 2 (b) $-(\mathrm{c})$.

Addressing the issue of inter-patient variability, the individualization of both model order and model parameters did not significantly increase the performance of the estimation as shown in Fig. 3. Statistically, the generalized model can be assumed to adequately describe all individuals considered, with no significant loss in accuracy. Thus, the generalized model identified proved to be robust regarding inter-patient variability. A close look at simulation results (Fig. 4 and 5) shows that that performance could be further increased by a more precise estimation of the variation entity. The model proves to be accurate in identifying the occurrence instants of fast variation in MAP, but less accurate in estimating their amplitude. A major improvement would be represented by including in the estimation procedure a further additional signal covering this remaining lack of information.

\section{CONCLUSIONS}

We have presented an effective procedure to continuously on-line estimate mean arterial blood pressure by means of HR and non-invasive intermittent measurements. The method was proven to be accurate and robust concerning inter-patient variability. The model could be integrated in the anaesthesia display to provide the anaesthetist with important additional information on the hemodynamic state of the patient when no continuous measurement of blood pressure is available. Further, the obtained improvement in the monitoring technique for MAP represents a key step in the development of blood pressure automatic control, allowing for using automatic MAP control also in the standard clinical cases of non-invasive blood pressure measurements.

\section{REFERENCES}

Allen, J. and A. Murray (1999). Modelling the relationship between peripheral blood pressure and blood volume pulses using linear and neural network system identification techniques. Physiol. Meas. 20, 287-301. 
Chen, W., T. Kobayashi, S. Ichikawa, Y. Takeuchi and T. Togawa (2000). Continuous estimation of systolic blood pressure using the pulse arrival time and intermittent calibration. Med. Biol. Eng. Comp. 38, 569-574.

Gentilini, A., C. Schaniel, M. Morari, C. Bieniok, R. Wymann and T. Schnider (2002). A new paradigm for the closed-loop intraoperative administration of analgesics in humans. IEEE Trans. Biom. Eng. 49, 289-299.

Gentilini, A., M. Rossoni, C. Frei, R. Wymann, M. Morari, A. Zbinden and T. Schnider (2001). Modelling and closed-loop control of hypnosis by means of bispectral index (bis) with isoflurane back to survey. IEEE Trans. Biom. Eng. 48, 874-889.

Gulcur, H. O. and Y. Bahadirlar (1998). Estimation of systolic blood pressure from the second heart sound. 2nd Int. Biom. Eng. Days pp. 39-41.

Habibi, S. and D. Coursin (1996). Assessment of sedation, analgesia, and neuromuscular blockade in peri-operative period. Int. Anesth. Clinics 34, 215-241.

Hürzeler, M. (2004). Zeitreihenanalyse. ETH Zürich, Nachdiplomkurs in Angewandter Statistik. Zürich.

Ljung, L. (1999). System Identification - Theory for the user. Prentice-Hall. New Jersey.

Mackay, R. S. (1964). The application of physical transducer to intracavity measurement, with special application to tonometry. Med. El. Biol. Eng. 2, 3-17.

Petersen-Felix, S., L. Arendt-Nielsen and A. M. Zbinden (1998). Do results from experimental nociceptive models reflect pain perception during general anaesthesia?. Pain Forum $7,43-45$.

Pinna, G. D., R. Maestre and A. Mortara (1996). Estimation of arterial blood pressure variability by spectral analysis: Comparison between finapres and invasive measurements. Physiol. Meas. 17, 147-169.

Prys-Roberts, C., J. W. Lloyd, A. Fisher, J. H. Kerr and T. J. S. Patterson (1990). Selftuning adaptive control of induced hypotension in humans: a comparison of isoflurane and sodium nitroprusside. J. Clin. Monit. 6, 236-240.

Rampil, I. J. (1998). A primer for eeg signal processing analysis. Anesthsiology 89, 9801002.

Stadler, K. S., D. Leibundgut, P. M. Schumacher, T. Bouillon, A. H. Glattfelder and A. M. Zbinden (2003). Modelling and closed-loop control of skeletal muscle relaxation during general anaesthesia using mivacurium back to survey. Proc. Europ. Control Conf. (ECC), Cambridge, UK.
Yamakoshi, K., H. Shimazy and T. Togawa (1980). Indirect measurement of instantaneous arterial blood pressure in the human finger by the vascular unloading technique. IEEE Trans. Biomed. Eng. pp. 150-155.

\section{APPENDIX}

The data set used for the analysis was obtained from a volunteer study designed to explore the effects of the hypnotic isoflurane and the analgesic alfentanil on several clinical end-points and to determine the reactions of such end-points to experimental painful stimuli.

After local ethics committee approval, 15 male and 5 female consenting healthy volunteers of physical status ASA I were enrolled for the study. From each subject, written informed consent was obtained. The age of the group considered ranged from 21 to 33 years, with an average value of 25 years. In addition to standard monitoring and bispectral index monitoring (Rampil, 1998), the neuromuscular activity of the volunteers was monitored by stimulation electrodes over the ulnar nerve. Arterial blood pressure was invasively measured with a catheter cannula inserted into the arteria radialis. All parameters were recorded using a Labview-based data acquisition system for offline analysis. General anaesthesia was induced by the single breath technique with a mixture of $7 \%$ sevoflurane in $60 \% \mathrm{~N}_{2} \mathrm{O}$ and maintained by continuous administration of isoflurane in $\mathrm{O}_{2}$. Bolus doses $(0.15 \mathrm{mg} / \mathrm{kg}$ body weight) of mivacurium were administered after loss of eyelid reflexes to facilitate intubation.

The subjects were randomly assigned to two groups receiving either fixed (predetermined) concentrations of alfentanil and stepwise varying concentrations of isoflurane or vice versa. Targeting infusion policies algorithms maintained a constant level of predicted drug concentration in the plasma. Each volunteer was kept at seven different combinations of hypnotic and analgesic, ranging between $0-400 \mathrm{ng} / \mathrm{ml}$ of alfentanil and $0-2.3 \%$ of isoflurane. Within each phase, after reaching steady state conditions, a series of experimental pain stimuli was applied in a randomized sequence. Experimental stimulation applied consisted of laryngoscopy, intubation, icewater-test, trapezius squeeze, electrical tetanic stimulation of the ulnar nerve $(50 \mathrm{~Hz}, 60 \mathrm{~mA}, 5 \mathrm{sec})$ and auditory evoked potential. 